\title{
Low-energy electron diffraction crystallography of surfaces and interfaces
}

Article

Published Version

Held, G. (2010) Low-energy electron diffraction crystallography of surfaces and interfaces. Bunsen-Magazin 12 (12). pp. 124131. Available at https://centaur.reading.ac.uk/15540/

It is advisable to refer to the publisher's version if you intend to cite from the work. See Guidance on citing.

Publisher: DBG Deutsche Bunsen-Gesellschaft für Physikalische Chemie e.V.

All outputs in CentAUR are protected by Intellectual Property Rights law, including copyright law. Copyright and IPR is retained by the creators or other copyright holders. Terms and conditions for use of this material are defined in the End User Agreement.

\section{www.reading.ac.uk/centaur}

\section{CentAUR}

Central Archive at the University of Reading

Reading's research outputs online 


\section{LOW-ENERGY ELECTRON DIFFRACTION CRYSTALLOGRAPHY OF SURFACES AND INTERFACES}

\section{METHOD SUMMARY}

\section{Acronyms, Synonyms \\ Low energy electron diffraction (LEED) \\ Micro channel plate (MCP) \\ Retarding Field Analyzer (RFA) \\ Intensity vs Voltage curves (IV curves) \\ Intensity vs Energy curves (I(E) curves)}

\section{Information available}

Technique is surface-sensitive.

Periodicity of surface layers (superstructure) - LEED pattern.

Degree of surface order (e.g. phase transitions, island size) spot profiles of LEED pattern.

Positions of atoms ( $\pm 1-10 \mathrm{pm}$ ) in the layers near the surface $(<1 \mathrm{~nm})$ - LEED-IV structure determination.

\section{Information not available (limitations)}

Not element-specific.

No information about bulk structure ( $>1 \mathrm{~nm}$ below surface). Requires long-range order (limited information about amorphous or random surface structures)

\section{INTRODUCTION}

When Clinton Davisson and Lester Germer conducted the very first low-energy electron diffraction (LEED) experiments in April 1925 at Bell Labs in New York it hit them - quite literally - like a lightening stroke: "At that time we were continuing an investigation ... of the distribution in-angle of electrons scattered by a target of ordinary nickel. During the course of this work a liquid-air bottle exploded at a time when the target was at high temperature; the experimental tube was broken, and the target heavily oxidized by the inrushing air. The oxide was eventually reduced and a layer of the target removed by vaporization but only after prolonged heating at various high temperatures in hydrogen and in vacuum. When the experiments were contin- ued it was found that the distribution-in-angle of the scattered electrons had been completely changed." [Davi27] They added, "We must admit that the results obtained in these experiments have proved to be quite at variance with our expectations."

The prolonged heating treatment had transformed the crystallites of the polycrystalline nickel sample into $\mathrm{mm}$ size crystals and the intensity distribution of elastically back-scattered electrons now showed sharp maxima instead of the smooth angular distribution before the accident. Davisson and Germer soon realized that these were interference patterns and, thus, the first experimental proof of the wave nature of electrons, which had been postulated only a few years before, in 1923, by Louis De Broglie. He had suggested that electrons have a wave length, which is proportional to the inverse of their momentum $m_{\mathrm{e}} \mathrm{v}$ :

$$
\lambda_{\mathrm{e}}=\mathrm{h} /\left(\mathrm{m}_{\mathrm{e}} \mathrm{v}\right)=\left(1.50 \mathrm{eV} / \mathrm{E}_{\mathrm{kin}}\right)^{1 / 2}[\mathrm{in} \mathrm{nm}]
$$

and a wave vector of length

$$
\mathrm{k}_{\mathrm{e}}=2 \pi / \lambda_{\mathrm{e}}=(2 \pi / \mathrm{h}) \cdot \mathrm{m}_{\mathrm{e}} \mathrm{v}
$$

which is proportional to the momentum of the electron ( $\mathrm{h}$ is Planck's constant, $m_{e}$ the electron mass, $v$ the velocity, and $E_{k i n}$ the kinetic energy of the electron). For low kinetic energies between a few ten and a few hundred electron volts $(\mathrm{eV})$ the wavelength is of the order of $0.1 \mathrm{~nm}$, i.e. comparable to typical interatomic distances in crystals and molecules and it was soon realized that the angular interference patterns observed in lowenergy electron diffraction (LEED) can be used to determine the structure of well-ordered crystals, in analogy to X-ray diffraction. Due to the small inelastic mean free path of electrons in this energy range, typically around $1 \mathrm{~nm}$, LEED samples only the topmost atomic layers of a crystal and is, therefore, best suited for the analysis of surface geometries. X-ray photons, on the other hand, have a much larger mean free path, typically a few $\mu \mathrm{m}$. Therefore X-ray diffraction delivers crystallographic information about the bulk-structure of a crystal. Another important difference is that multiple scattering plays an important role in the diffraction process of electrons at solid surfaces, which is not the case for photons. Therefore, the analysis of LEED data with

\section{Dr. Georg Held}

Department of Chemistry, University of Reading

Whiteknights Reading RG6 6AD

United Kingdom

Tel.: +44 (0)118 378 6347, Fax: +44 (0)118 3786331

E-Mail: g.held@reading.ac.uk 
respect to the exact positions of atoms at a surface is somewhat more complicated and requires fully dynamical quantum mechanical scattering calculations.

The use of LEED as a standard technique for surface analysis started in the early 1960's when large enough single crystals and commercial instruments became available for surface studies. At first the technique was only used for qualitative characterization of surface ordering and the identification of two-dimensional superstructures. The quantitative information about the positions of the atoms within the surface is hidden in the energy-dependence of the diffraction spot intensities, the so-called LEED I-V, or I(E), curves. Computer programs and the computer power to analyze these data became available in the 1970's. With the ever growing speed of modern computers LEED-IV structure determination has been applied to increasingly complex surface structures. To date LEED is the most precise and versatile technique for surface crystallography.

For further information about the history, experimental setup, and theoretical approaches of LEED refer to the books by Pendry, [Pend74], Van Hove and Tong [Vanh79], Van Hove, Weinberg and Chan [Vanh86], and Clarke [Clar85]. The present article makes extensive use of these works.

\section{BASIC PRINCIPLES}

The basic principle of a standard LEED experiment is very simple: a collimated mono-energetic beam of electrons is directed towards a single crystal surface and the diffraction pattern of the elastically back-scattered electrons is recorded using a position-sensitive detector. For electrons, like for all wave-like objects, the angular intensity distribution due to the interference of partial waves back-scattered from a periodic array is described by Bragg's law or, more conveniently, by a set of Laue equations, one for each dimension of periodicity, which predict a regular pattern of diffraction spots.

\subsection{SURFACE PERIODICITY AND RECIPROCAL LATTICE}

Because of the short penetration depth of low-energy electrons the diffraction process is determined by a small number atomic layers at the crystal surface. The electrons do not probe the full crystal periodicity perpendicular to the surface. Therefore, the array of relevant scatterers is only periodic in two dimensions. The surface lattice can be described by a pair of lattice vectors $\mathbf{a}_{\mathbf{1}}$ and $\mathbf{a}_{\mathbf{2}}$, which are parallel to the surface plane, and the surface unit cell, i.e. the contents of the parallelogram spanned by $\mathbf{a}_{\mathbf{1}}$ and $\mathbf{a}_{\mathbf{2}}$. The surface consists of identical copies of the unit cell at every point

$$
\mathbf{R}=m_{1} \mathbf{a}_{\mathbf{1}}+m_{2} \mathbf{a}_{\mathbf{2}}
$$

with integer numbers $m_{1}$ and $m_{2}$. The left hand side of Figure 1 illustrates common square, rectangular and hexagonal surfaces and the lattice vectors defining their unit cells.

The two-dimensional Laue equations are based reciprocal lattice vectors within the surface plane which are defined by the real space lattice vectors through a set of four simultaneous equations:
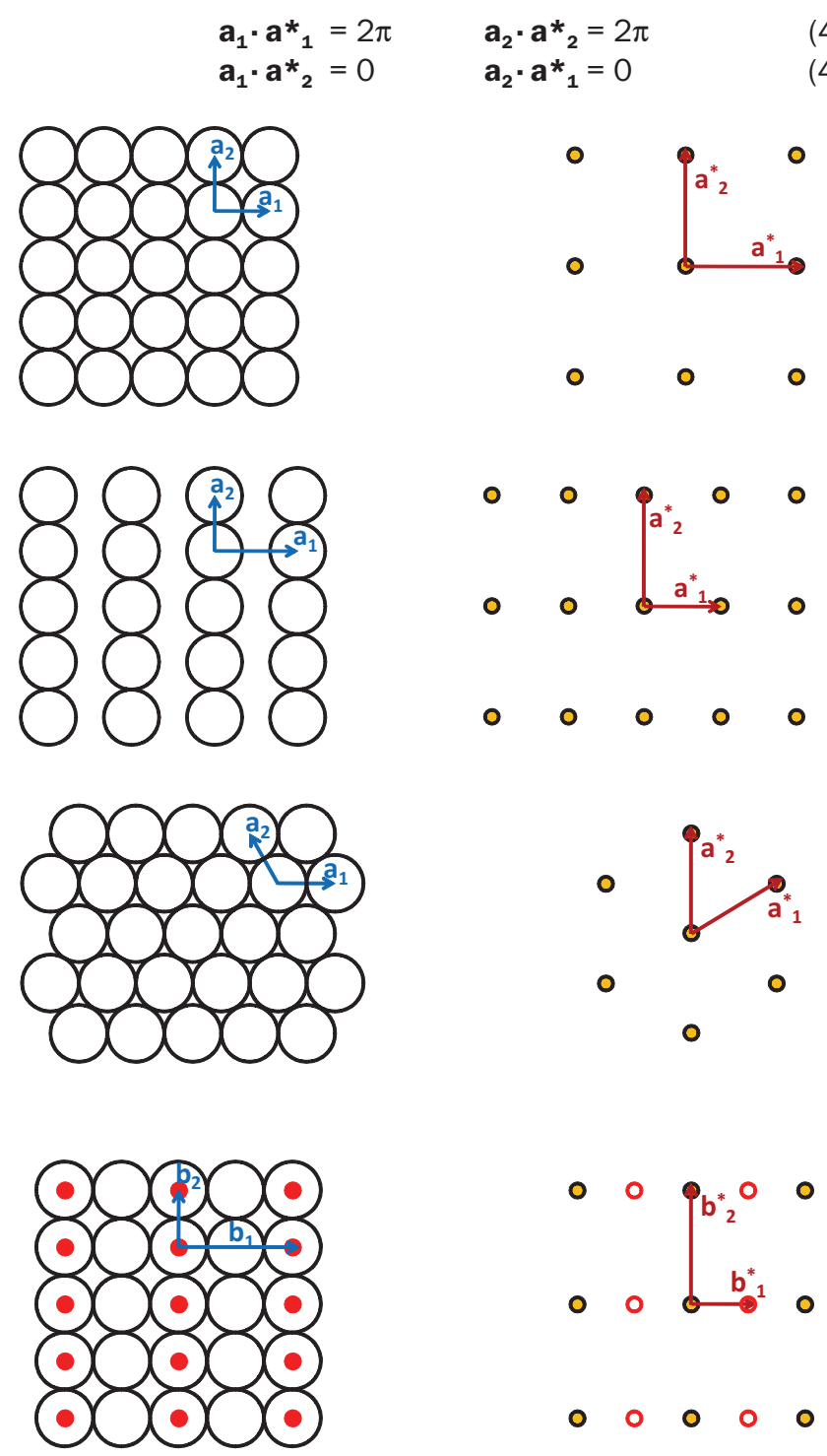

Figure 1 (left from top to bottom) arrangement of atoms in the $\{100\}$ (square) \{110\} (rectangular) and $\{111\}$ (hexagonal) surfaces of a simple face centered cubic crystal lattice and a p(2x1) superstructure on a square surface; the diagrams include lattice vectors defining the surface unit cell and the corresponding reciprocal lattices (right).

In order for the scalar products in (4a) to be dimensionless, the reciprocal lattice vectors must have units of inverse length, $\mathrm{nm}^{-1}$. As a consequence of $(4 \mathrm{~b}) \mathbf{a}{ }^{*}$ and $\mathbf{a}{ }^{*}{ }_{1}$ must be perpendicular to $\mathbf{a}_{\mathbf{1}}$ and $\mathbf{a}_{\mathbf{2}}$, respectively, which means that a rectangular realspace lattice will also have a rectangular reciprocal lattice. For non-rectangular lattices the angles are different in real space and reciprocal space. The right-hand column of Figure 1 shows the corresponding reciprocal lattices for each of the surfaces on the left. The reciprocal lattice vectors define the positions of the diffraction maxima through the Laue equation (5).

$$
\mathbf{k}_{\mathrm{Il}, \text { out }}\left(\mathrm{n}_{1}, \mathrm{n}_{2}\right)=\mathbf{k}_{\mathrm{ll}, \text { in }}+\mathrm{n}_{1} \mathbf{a} \boldsymbol{*}_{\mathbf{1}}+\mathrm{n}_{2} \mathbf{a} \boldsymbol{*}_{\mathbf{2}}
$$

$\mathbf{k}_{\mathrm{Il}, \text { out }}$ is the component of the wave vector of the diffracted electrons, which is parallel to the surface plane (by conven- 
tion, this is the $x y$-plane). $\mathbf{k}_{\mathrm{Il}, \text { in }}$ is the parallel component of the wave vector of the incoming electron beam. Note that the Laue equation (5) defines a two-dimensional vector, hence it actually comprises two equations, one for each component. Each diffraction spot corresponds to the sum of integer multiples of $\mathbf{a}{ }_{1}$ and $\mathbf{a}{ }_{2}$. The integer numbers $\left(n_{1}, n_{2}\right)$ are used as indices to label the spots.

Energy conservation demands that the length of the $\mathbf{k}$-vector is the same, $\left(2 m_{e} E_{k i n} / h^{2}\right)^{1 / 2}$, for both the incoming and the elastically scattered electron wave. This defines the vertical or $z$ component, $k_{z, \text { out }}$, of the back-diffracted electrons in the $\left(n_{1}, n_{2}\right)$ spot:

$$
\mathrm{k}_{\mathrm{z}, \text { out }}\left(\mathrm{n}_{1}, \mathrm{n}_{2}\right)=\left[2 \mathrm{~m}_{\mathrm{e}} \mathrm{E}_{\mathrm{kin}} / \mathrm{h}^{2}-\left|\mathbf{k}_{\mathrm{ll}, \text { out }}\left(\mathrm{n}_{1}, \mathrm{n}_{2}\right)\right|^{2}\right]^{1 / 2}
$$

Note that, unlike for X-ray diffraction, there is no Laue-condition for the z-component of $\mathbf{k}_{\text {out }}$. The only condition for diffraction into a spot $\left(n_{1}, n_{2}\right)$ is that $k_{z, \text { out }}$ has a real value, i.e. the argument of the square root ( [ $]^{1 / 2}$ ) on the right hand side of equation (6) must not be less than zero. This condition is synonymous with the obvious fact that the length of the parallel component of $\mathbf{k}$ cannot be greater than the length of the entire vector, but it also limits the number of observable LEED spots. The number of observable spots increases With increasing electron energy while the polar emission angle with respect to the specular spot $(0,0)$ decreases for each spot. This is illustrated in Figure 2 for normal incidence $\left(\mathbf{k}_{\mathrm{Il} \text {,in }}=0\right)$; in this case $\mathbf{k}_{\mathrm{Il} \text {,out }}\left(\mathrm{n}_{1}, \mathrm{n}_{2}\right)=$ $\mathrm{n}_{1} \mathbf{a}{ }_{1}+\mathrm{n}_{2} \mathbf{a} \boldsymbol{*}_{\mathbf{2}}$ is constant for a given pair of spot indices and only $\mathrm{k}_{\mathrm{z} \text { out }}$ is affected by changes in the kinetic energy.

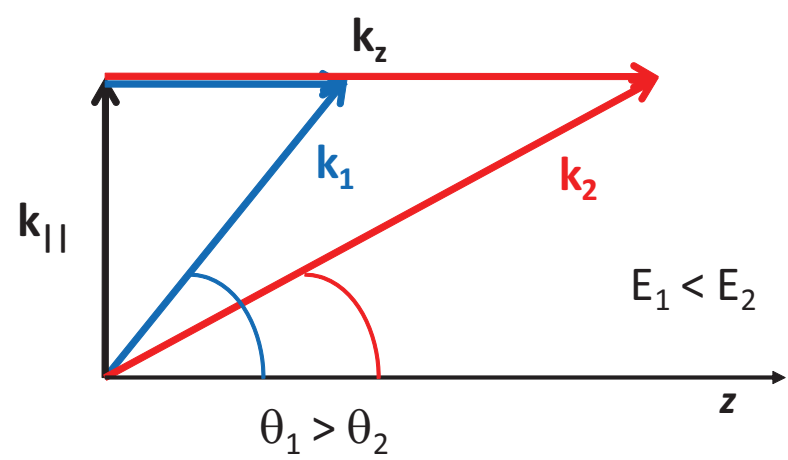

Figure 2: Relationship between $k_{2}, k_{1}$ and the emission angle for a diffracted electron wave at two different energies.

Only the specular spot does not change its position as a function of energy if the angle of incidence is kept constant.

\subsection{SUPERSTRUCTURES}

Superstructures fromed by adsorbates or rearrangements of the surface atoms can lead to a periodicity of the surface lattice greater than that of the bulk-truncated single crystal. In these cases, the lattice vectors for the superstructure, $\mathbf{b}_{1}$ and $\mathbf{b}_{\mathbf{2}}$, can always be related to the lattice vectors of the bulk-truncated surface, $\mathbf{a}_{\mathbf{1}}$ and $\mathbf{a}_{\mathbf{2}}$, through

$$
\begin{aligned}
& \mathbf{b}_{\mathbf{1}}=\mathrm{m}_{11} \mathbf{a}_{\mathbf{1}}+\mathrm{m}_{12} \mathbf{a}_{\mathbf{2}} \\
& \mathbf{b}_{\mathbf{2}}=\mathrm{m}_{21} \mathbf{a}_{\mathbf{1}}+\mathrm{m}_{22} \mathbf{a}_{\mathbf{2}}
\end{aligned}
$$

the numbers $m_{i j}$ are the coefficients of the superstructure matrix $M=\left[m_{11} m_{12} ; m_{21} m_{22}\right]$, which is a straightforward way of characterising any superstructure. Depending on whether all $m_{i j}$ are interger numbers or not the superstructure is either called commensurate or incommensurate. Superstructures lead to additional spots in the LEED pattern, for which fractional indices are used. The reciprocal lattice vectors for these spots can be calculated directly from the coefficients of the superstructure matrix according to the following set of equations [Vanh86]:

$$
\begin{aligned}
& \mathbf{b}{ }_{1}=\left(m_{11} \cdot m_{22}-m_{12} \cdot m_{21}\right)^{-1} \cdot\left(m_{22} \mathbf{a}{ }_{1}-m_{21} \mathbf{a}{ }^{*}\right) \\
& \mathbf{b}^{*}{ }_{1}=\left(m_{11} \cdot m_{22}-m_{12} \cdot m_{21}\right)^{-1} \cdot\left(-m_{12} \mathbf{a}{ }_{1}-m_{11} \mathbf{a}{ }_{2}\right)
\end{aligned}
$$

The fractional indices of the superstructure spots are multiples of the prefactors of $\mathbf{a}{ }_{1}$ and $\mathbf{a}{ }_{2}$ in equations (6).

Another, less general notation according to Wood [Wood64] specifies the lengths of the vectors $\mathbf{b}_{\mathbf{1}}$ and $\mathbf{b}_{\mathbf{2}}$ in units of $\mathbf{a}_{\mathbf{1}}$ and $\mathbf{a}_{\mathbf{2}}$, respectively, together with the rotation angle $\alpha$ between $\mathbf{b}_{1}$ and $\mathbf{a}_{\mathbf{1}}$ (only specified if $\alpha$ is not zero):

$$
p / c\left(\left|\mathbf{b}_{\mathbf{1}}\right| /\left|\mathbf{a}_{\mathbf{1}}\right| \times\left|\mathbf{b}_{\mathbf{2}}\right| /\left|\mathbf{a}_{\mathbf{1}}\right|\right) R \alpha
$$

$p$ indicates a "primitive" and $c$ a "centred" surface unit cell. Examples are " $p(2 \times 1)$ ", " $p(\sqrt{ } 3 \times \sqrt{ } 3) R 30^{\circ}$ ", and " $c(2 \times 2)$ ". This notation is not applicable to all superstructures but it is more frequently used than the matrix notation because it is shorter. As an example, a $p(2 \times 1)$ superstructure on a square substrate surface is shown at the bottom of Figure 1 . The corresponding superstructure matrix is [2 $0 ; 0$ 1] and the reciprocal lattive vectors are $\mathbf{b}{ }_{1}=1 / 2 \cdot \mathbf{a}{ }_{1}$ and $\mathbf{b}{ }_{\mathbf{2}}=\mathbf{a}{ }_{\mathbf{2}}$.

\subsection{SPOT INTENSITY VS ENERGY}

There is no Laue-condition for the $z$ component of $\mathbf{k}_{\text {out }}$, i.e. diffraction spots are allowed for a wide range of kinetic energies. This does not mean, however, that the intensities of spots are constant with the energy. Although the electrons do not experience the full periodicity of the crystal perpendicular to the surface, there is still interference of electrons scattered from different atomic layers parallel to the surface. For infinite penetration depth this would impose a third Laue condition for $\mathrm{k}_{\mathrm{z}, \mathrm{out}}$ and therefore each $\left(\mathrm{n}_{1}, \mathrm{n}_{2}\right)$ spot would have sharp intensity maxima ("Bragg peaks") for certain values of $E_{k i n}$ (cf equation (6) ) and zero intensity for all other energies. Since the penetration depth is very small, the back-scattered electrons only interact with a few layers of atoms giving rise to broad maxima at the Bragg peak positions and non-zero intensities in the intermediate energy regimes of the intensity vs energy curve of each spot (also known as intensity vs voltage or IV curve). The combination of non-periodic layer distances near the surface, different atomic scattering potentials and multiple scattering events leads to shifts in the Bragg peaks and intensity maxima at other energies in the IV curves. All these effects are reproduced by fully dynamical quantum mechanical scattering calculations [Pend74,Vanh79]. An example is given in Figure 7. 


\subsection{SPOT PROFILES}

While the spot positions and intensities carry information about the size and the local geometry within the surface unit cell, the spot profile, i.e. the shape and width of a diffraction spot, is determined by the long range relative arrangement the unit cells at the surface. Vertical displacements of the surface unit cells (e.g. steps, facets) lead to split spots and changes of the spot profile as a function of electron energy. If all surface unit cells are in the same plane (over a length of at least 10nm, which is a typical coherence width of LEED instruments), the spot profile does not change with energy.

A periodic arrangement of equal steps at the surface causes spot splitting at energies, which lead to destructive interference between electrons reflected from adjacent terraces ("outof-phase condition"). By measuring these energies the step height can be determined directly. For a more random arrangement of steps the analysis of energy dependent changes in the spot profiles allows in many cases the determination of the mean step height and a characterization of the step distribution [Henz77,Woll98]. Facets lead to extra spots which move in $\mathbf{k}_{\mathrm{II}}$ upon changes of the kinetic energy.

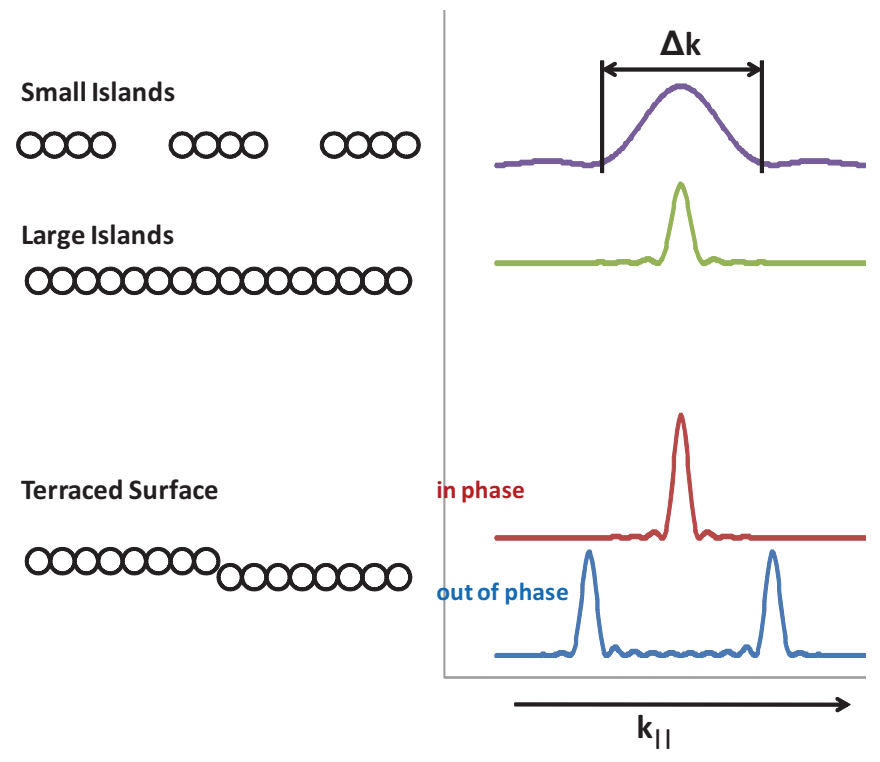

Figure 3: Effect of island size on the spot profile (top) and spot splitting induced by regular steps (bottom); in phase: constructive interference between electrons reflected from adjacent terraces; out of phase: destructive interference. (According to [Henz91])

Point defects, static disorder, and thermally induced displacements lead to an increase of the background intensity between the spots. Depending on the correlation between the scatterers, the background is either homogeneous (no correlation) or structured (correlation). If the coherently ordered surface areas (islands, domains) are small $(<10 \mathrm{~nm})$ and at the same vertical height, the width of these areas, $\Delta \mathrm{w}$, is inversely proportional to the width of the LEED spots, $\left|\Delta \mathbf{k}_{1 \mid}\right|$ :

$$
\left|\Delta \mathbf{k}_{\mathrm{II}}\right|=2 \pi / \Delta \mathrm{w}
$$

This relation holds for each direction parallel to the surface independently. It is particularly useful for determining the size of adsorbate islands which lead to extra superstructure spots. A good introduction (in German) into spot profile analysis is given in the book by Henzler and Göpel [Henz91].

\section{EXPERIMENT}

The standard modern LEED system is of the "rear view" type, which is schematically depicted in Figure 4. The incident electron beam, accelerated by the potential $V_{0}$, is emitted from the electron gun behind a transparent hemispherical fluorescent screen and hits the sample through a hole in the screen. Typically, the electron beam has a current of around $1 \mu \mathrm{A}$ and a diameter of 0.5 to $1 \mathrm{~mm}$. The surface is in the centre of the hemisphere so that all back-diffracted electrons travel towards the LEED screen on radial trajectories.

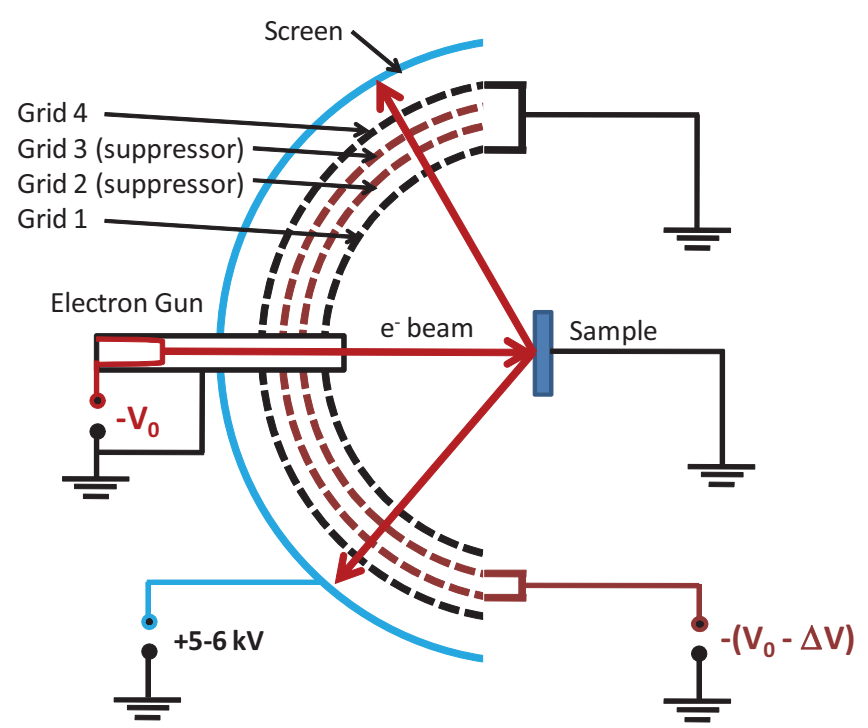

Figure 4: Schematic diagram of a typical LEED instrument.

Before the electrons hit the screen they have to pass a retarding field energy analyzer (RFA). It consists of four (sometimes three) hemispherical grids concentric with the screen, each containing a central hole, through which the electron gun is inserted. The first grid (nearest to the sample) is connected to earth ground as is the sample, in order to provide a field-free region between the sample and this grid. A negative potential $-\left(\mathrm{V}_{0}-\Delta \mathrm{V}\right)$ is applied to the second and third grid, the so-called suppressor grids. These repel all electrons that have undergone non-elastic scattering processes and have lost more than $\mathrm{e} \Delta \mathrm{V}$ (typically around $5 \mathrm{eV}$ ) of their original kinetic energy. Thus, only elastically scattered electrons and those with small energy losses can pass through to the fluorescent screen. The fourth grid is usually on ground potential in order to reduce field penetration of the screen voltage to the suppressor grids. The screen is at a potential of the order of 5-6 kV; it provides the electrons with enough energy to make the diffraction pattern visible on the fluorescent screen. The pattern can be observed through a view-port from behind the transparent screen. Only the electron gun assembly (diameter $<15 \mathrm{~mm}$ ) limits the view slightly.

MCP-LEED systems with position sensitive "micro channel plate" (MCP) electron multipliers between the RFA grids and 
the fluorescent screen have become commercially available in recent years for applications that require low incident beam currents, either to avoid beam damage (e.g. organic molecules) or charging of insulating samples (e.g. oxides). These systems can be operated with electron currents as low as $1 \mathrm{nA}$. Typical LEED systems have diameters of around $140 \mathrm{~mm}$.

The LEED pattern is recorded using a video camera with suitable image processing software. As with all methods that use electrons as probes, vacuum conditions are required because electrons cannot penetrate a gas atmosphere at normal pressures. In general, however, the vacuum conditions required to avoid contamination of clean surfaces are more rigorous (typically $<10^{-9}$ mbar) than those imposed by the use of electrons (typically $<10^{-6} \mathrm{mbar}$ ).

\section{APPLICATIONS}

In this section we will discuss a small selection of typical applications of LEED in order to illustrate the different levels at which this technique yields information about surface geometries.

\subsection{LEED PATTERN: CO ON NI\{111\}}

The adsorption of carbon monoxide on the $\{111\}$ surface of nickel is a good example how LEED diffraction patterns can be used for a simple characterization of adsorbate structures. With increasing coverage of $\mathrm{CO}$ adsorbed on $\mathrm{Ni}\{111\}$ four different LEED patterns are observed between about 0.30 and 0.62ML (1 ML corresponds to 1 molecule per substrate surface atom):

- a diffuse $\left[21 ;-11\right.$ ] or $p(\sqrt{ } 3 \times \sqrt{ } 3) R 30^{\circ}$ pattern between 0.3 and $0.4 \mathrm{ML}$,

- a sharp [2 0; 12 ] or $c(2 \times 4)$ pattern for coverage around $0.5 \mathrm{ML}$,

- a sharp [3 1; -1 2] or $p(\sqrt{ } 7 \times \sqrt{ } 7) R 19^{\circ}$ pattern between 0.56 and $0.60 \mathrm{ML}$,

- a more complicated [3 $2 ;-12$ ] pattern at the maximum coverage of $0.62 \mathrm{ML}$, which is described as " $c(2 \sqrt{3} \times 4) r e c t$ " in non-standard Wood notation.

Images of the first three patterns are depicted in Figure 5 together with the corresponding real-space unit cells (red arrows and dashed lines). The middle part of the Figure also shows the complete $(2 \times 4)$ unit cell (in black). Note that the " $c$ " in the Wood notation $c(2 \times 4)$ means that the center and the corners of the $(2 \times 4)$ unit cell are lattice points. Therefore the primitive unit cell is only half the size, as indicated by the red arrows. The matrix notation always refers to the primitive unit cell. The yellow arrows in the LEED patterns (left) indicate the reciprocal lattice vectors corresponding to the unit cells marked in red.

For the $c(2 \times 4)$ and $p(\sqrt{ } 7 \times \sqrt{ } 7) R 19^{\circ}$ structures it is not possible to reach all diffraction spots by adding integer multiples of these two vectors. This is because the observed pattern is a superposition of LEED patterns arising from different parts
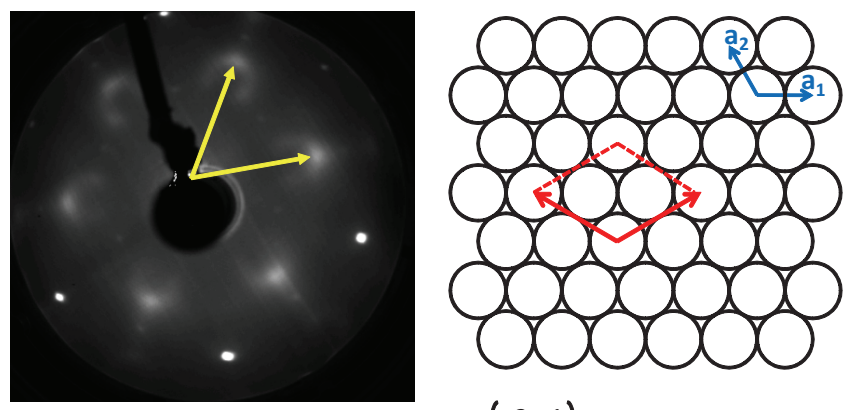

$\Theta=0.33 \mathrm{ML}: \quad \mathrm{p}(\sqrt{ } 3 \times \sqrt{ } 3) \mathrm{R} 30^{\circ}$ $M=\left(\begin{array}{rr}2 & 1 \\ -1 & 1\end{array}\right)$
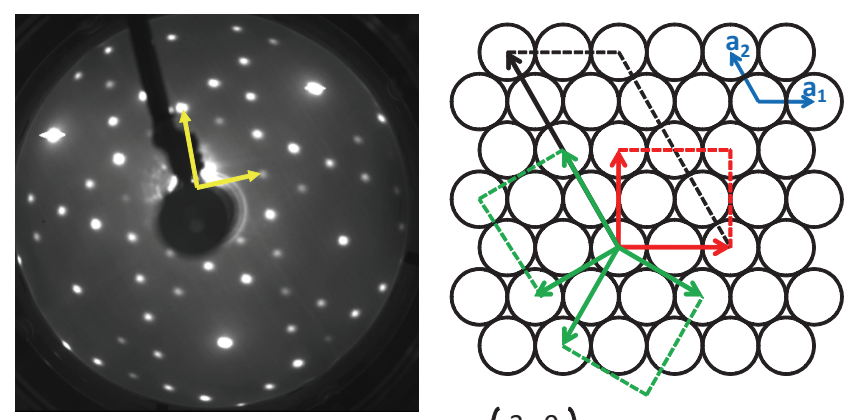

$\Theta=0.50 \mathrm{ML}: \quad c(2 \times 4)$ $M=\left(\begin{array}{ll}2 & 0 \\ 1 & 2\end{array}\right)$
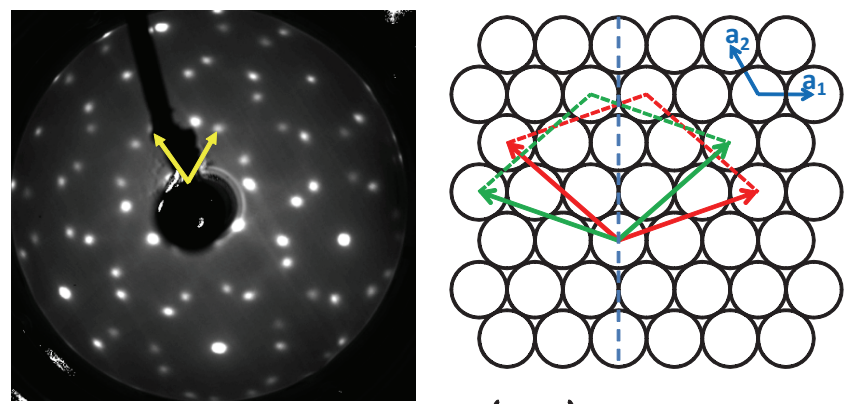

$\Theta=0.57 \mathrm{ML}: \quad \mathrm{p}(\sqrt{ } 7 \times \sqrt{ } 7) \mathrm{R} 19^{\circ}$ $M=\left(\begin{array}{rr}3 & 1 \\ -1 & 2\end{array}\right)$

Figure 5: Experimental LEED patterns formed by $\mathrm{CO}$ adsorbed on $\mathrm{Ni}\{111\}$ (left) and corresponding real-space unit cells (right): $\mathbf{p}(\sqrt{ } \mathbf{3} \times \sqrt{ } \mathbf{3}) \mathbf{R}^{\circ} 0^{\circ}$ (top, $\left.E_{\text {kin }}=98 \mathrm{eV}\right) c(2 \times 4)\left(\right.$ middle, $\left.E_{\text {kin }}=129 \mathrm{eV}\right)$ and $p(\sqrt{7} \times \sqrt{7}) \mathrm{R}^{\circ} 9^{\circ}$ (bottom, $\mathrm{E}_{\mathrm{kin}}=$ $117 \mathrm{eV}$ ). Note that real space diagrams are rotated by about $30^{\circ}$ with respect to the crystal orientation of the experiment; the dark structure extending from the top left to the middle of the LEED patterns is the shadow of the electron gun [Held98].

of the surface, where the ordered arrangements of molecules are the same in principle but may have different orientations. Such rotation or mirror domains are usually observed if the superstructure has lower symmetry than the underlying substrate alone. Any symmetry operation of the substrate surface (rotation or mirror) that is not shared with the superstructure will therefore convert the superstructure unit cell into a unit cell that is equivalent but has a different orientation. This new unit cell has a different reciprocal lattice with a new set of diffraction spots. All orientation domains are equivalent and will, therefore, cover equal areas of the surface. In the case of the $c(2 \times 4)$ superstructure, which has a rectangular unit cell, the missing symmetry is the three-fold rotation of the hexagonal substrate surface; therefore there are two additional rotational 
domains, indicated in green, each of which gives rise to a separate set of diffraction spots. The $p(\sqrt{ } 7 \times \sqrt{ } 7) R 19^{\circ}$ superstructure has a three-fold rotation symmetry but does not share the mirror symmetry plane with the substrate (dashed line) this leads to an extra mirror domain, again indicated in green, with a set of extra diffraction spots.

If the adsorbate coverage is known from other methods, as in the present example, it is straight forward to work out the number of molecules per unit cell: there is one molecule in the $p(\sqrt{3} \times \sqrt{3}) R 30^{\circ}$ unit cell (coverage $\left.1 / 3\right)$, two in the $c(2 \times 4)$ (coverage $2 / 4)$ and four molecules in the $p(\sqrt{ } 7 \times \sqrt{ } 7) R 19^{\circ}$ unit cell (coverage 4/7).

The diffraction spots of the $p(\sqrt{3} \times \sqrt{3}) R 30^{\circ}$ pattern are significantly broader than those of the other structures. This indicates that the ordered domains are considerably smaller than the coherence or transfer width of the LEED system. The radial spot width is about $1 / 5$ of the length of the reciprocal lattice vectors, therefore the corresponding width of the domains is on average about five unit cells or $2 \mathrm{~nm}$.

\subsection{SPOT PROFILES}

Figure 6 shows an example of energy-dependent changes in the spot profiles of terraced surfaces [Woll98]. The data were collected from a vicinal $\operatorname{Pd}\{100\}$ surface, which is tilted by $1.1^{\circ}$ with respect to the (100) plane. This leads to terraces with (100) orientation, like in at the top of Figure 1, separated by steps parallel to the [011] direction (vector $\mathbf{a}_{\mathbf{1}}$ in the top diagram of Figure 1). The scan direction for the spot profiles is perpendicular to the step edges, i.e. along $\mathbf{a}_{\mathbf{2}}$ in real space or $\mathbf{a}_{\mathbf{2}}$ * in reciprocal space, respectively. The abscissa units of Figure 6 are percent fractions of $\left|\mathbf{a}_{\mathbf{2}}{ }^{*}\right|$. The parameter $S$ is a dimensionless quantity, which is proportional to $k_{z, \text { out }}\left(n_{1}, n_{2}\right)$ and, hence, depends on the electron energy through Equation (6). $S$ describes the phase difference between electron waves emerging from different terraces in a convenient way: an integer value of $S$ indicates the in-phase condition or constructive interference for all terraces, whereas an integer value plus 0.5 corresponds to maximum destructive interference (out-ofphase condition) between terraces separated by mono-atomic steps.

The spot profile changes very dramatically from a single sharp peak at the expected spot position ( 0 ) for $S=4.0$ to a double peak with a minimum at the actual spot position for $S=3.5$. The separation between the two peaks is $2.8 \%$ of $\left|\mathbf{a}_{\mathbf{2}}{ }^{*}\right|$, therefore the average terrace width in this direction is $(0.028)^{-1} \cdot\left|\mathbf{a}_{\mathbf{2}}\right|=$ $36\left|\mathbf{a}_{\mathbf{2}}\right|$ or $9.8 \mathrm{~nm}$, which is the value expected for a tilt angle of $1.1^{\circ}$. (Note that the factor $2 \pi$ in Equation (10) is not needed when $\left|\Delta \mathbf{k}_{||}\right|$and $\Delta \mathrm{w}$ are expressed as multiples of real and reciprocal lattice vectors.) By fitting the peak shape additional information about the width distribution and roughness of the surface can be obtained, which is described in detail by Wollschläger et al. in [Woll98].

Obviously, this kind of information can also be obtained by scanning probe microscopy (STM, AFM) with less sophisticated data analysis. The advantage of LEED spot profile analysis is that the data acquisition is fast and can easily be performed while the surface undergoes structural changes (e.g. varying temperature, during adsorption). LEED also provides an average over much larger surface areas (typically $1 \mathrm{~mm}^{2}$ ) than microscopic techniques can normally image simultaneously.

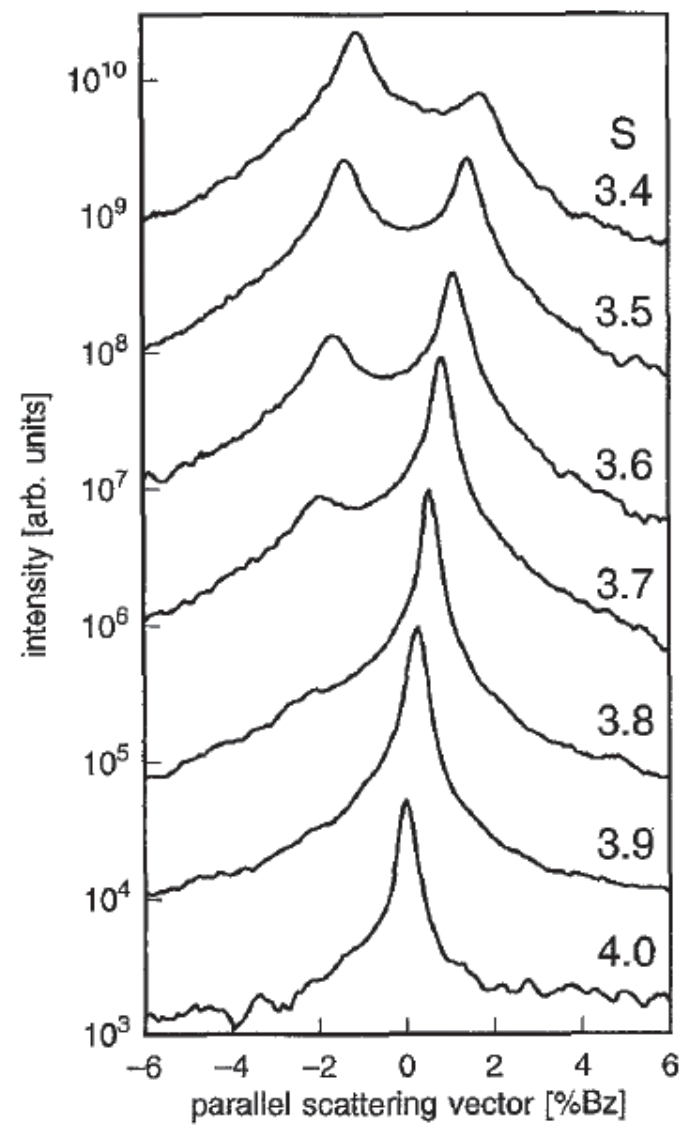

Figure 6: Profiles of the $(0,0)$ spot from a terraced (vicinal) $\operatorname{Pd}\{100\}$ surface recorded perpendicular to the step edges. The abscissa units are percent fractions of $\left|a_{2}{ }^{*}\right|$.

\subsection{LEED-IV STRUCTURE DETERMINATION}

As discussed in Section 2.3, the three-dimensional arrangement of atoms within the unit cell is responsible for the spotintensity variations as a function of electron energy, the LEEDIV curves. Modern electron scattering programs reproduce all features observed in LEED-IV curves, however, the dominance of multiple scattering in electron diffraction does normally not permit determining the surface geometry directly from a set of experimental IV curves. Instead, LEED-IV structure determination works on the principle of "trial and error". Theoretical IV curves are calculated for a large number of model geometries and compared with the corresponding experimental curves. The agreement is quantified by the means of a reliability factor or R-factor. There are several ways of defining such R-factors [Vanh86] with Pendry's R-factor, $R_{p}$, being the most common one [Pend80]. By convention, $R_{p}$ is 0 when the agreement is perfect and 1 for uncorrelated sets of IV curves. Usually, automated search procedures are used, which modify the model geometries until an R-factor minimum is found. The geometry with the lowest R-factor is the result of the structure determination. 

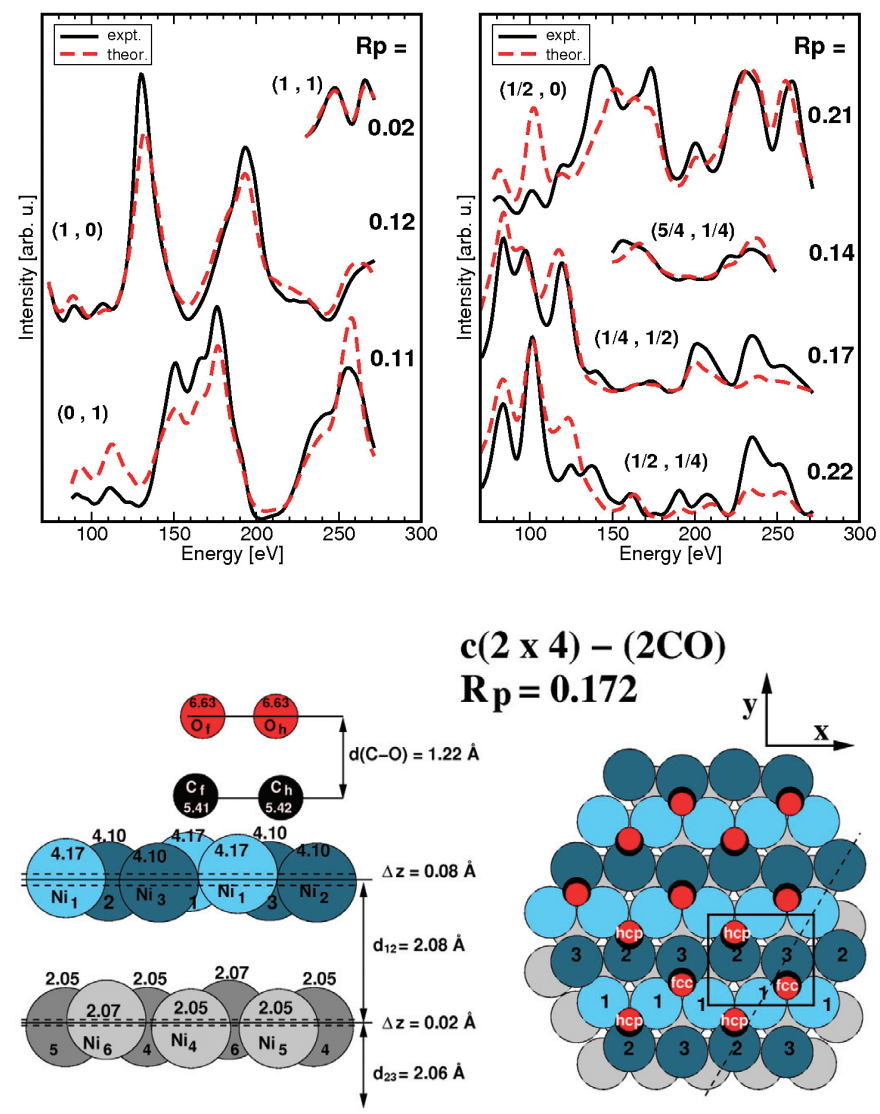

Figure 7: LEED-IV curves ( 70 to $270 \mathrm{eV}$ ) and resulting surface geometry of the $\mathrm{c}(2 \times 4)$ superstructure of $\mathrm{CO}$ on $\mathrm{Ni}\{111\}$ [Brau05a].

The level of precision in the resulting crystallographic data depends on the lowest R-factor achieved and the total energy range of overlapping experimental and theoretical IV curves. The energy overlap is typically between 1000 and $3000 \mathrm{eV}$, depending on the number of observable spots. Typically, $R_{p}$ values of around 0.1 can be expected for clean close packed metal surfaces, for more complex metal and semiconductor surfaces and adsorption structures of simple molecules one can reach $\mathrm{R}_{\mathrm{p}}$-factors of around 0.15 to 0.25 , and 0.25 to 0.35 for more complex molecular superstructures. The main reason for the gradually worse agreement between theoretical and experimental IV curves as the surface structures become more complex lies in the approximations in conventional LEED theory, which treat the atoms as perfect spheres with constant scattering potential in between ("muffin-tin potential"). This is description is somewhat inaccurate for the scattering potential of more open surfaces and organic molecules. As a consequence, a precision of $1-2 \mathrm{pm}$ for can be achieved for atoms in close packed metal surfaces, whereas the positions of atoms within organic molecules are typically determined within $\pm 10-20 \mathrm{pm}$. The coordinates perpendicular to the surface are usually more precise that those parallel to the surface plane, because the main scattering direction is perpendicular to the surface.

Examples of experimental and best-fit theoretical IV curves for one of the previous examples, the $c(2 \times 4)$ structure of $\mathrm{CO}$ on $\mathrm{Ni}\{111\}$, are shown at the top of Figure 7 [Brau05a]. The Graph also lists the individual R-factors for each pair of theoretical and experimental IV-curves. The geometry with the lowest average $\mathrm{R}$-factor, 0.172 (average of weighted with the energy range of each individual IV curve), is shown at the bottom of Figure 7. The unit cell contains two $\mathrm{CO}$ molecules adsorbed on two different three-fold hollow sites. The coordinates of the molecules and the first two layers of $\mathrm{Ni}$ atoms were determined within the structure analysis. The precision for the coordinates of the $\mathrm{Ni}$ atoms is between $3 \mathrm{pm}(z)$ and $9 \mathrm{pm}(x, y)$. Carbon and oxygen atoms are weak scatterers, therefore, their contribution to the intensity variations in the IV curves is smaller than that of the $\mathrm{Ni}$ atoms and consequently their coordinates are less precise, between $4 \mathrm{pm}(z)$ and $20 \mathrm{pm}(x, y)$.

Owing to the vast increase in available computer power, close to thousand surface structures have been determined in the last three decades, the majority of which were clean metal and semiconductor surfaces and adsorbate structures of atoms and small molecules. Two review articles by Heinz et al. [Hein94] and Over [Over98] provide good overviews and discussions of LEED structure determinations of clean and adsorbate-covered surfaces and further references. The "NIST Surface Structure Data Base" compiled by Watson et al. contains a complete list of all structures up to 2002 [SSD_02].

More recently, the capabilities of LEED-IV structure determination have been significantly extended to solve more complex surface structures, such as those of quasi-crystals [Ferr04], graphene overlayers [Mori10] and adsorption structures of important organic molecules such as benzene [Held01] and $\mathrm{C}_{60}$ [Li_09].

\subsection{LEED-IV ON DISORDERED LAYERS}

Usually, LEED-IV structure determination of layers of adsorbed atoms or molecules requires single crystal surfaces with longrange ordered adsorbate layers. Structural information for adsorbate-covered surfaces without long-range order can be obtained, however, in a similar way, when the energy dependence of the diffusely scattered intensity is analyzed (Diffuse LEED [Hein91,Hein92]) or from the IV curves of integer-order spots, which are still observed even if the adsorbate layer is not ordered [Poon04,Brau05b]. In both cases, however, the data analysis must assume that the local adsorption geometry is the same for all adsorbates. The main problem in both approaches is the amount of data (energy overlap) available for the analysis. This problem can be solved by recording data for different angles of incidence. If the difference in incidence angles is sufficient, each angle will provide an independent set of IV curves, which can greatly improve the reliability and precision of the structure determination [Held95].

\section{CONCLUSIONS AND PERSPECTIVES}

LEED is the most accurate and powerful experimental technique for surface crystallography at a level of precision that enables the chemical characterization of inter-atomic bonds. Often scanning probe microscopy (e.g. scanning tunneling microscopy, STM, atomic force microscopy, AFM) is seen as an alternative because it yields direct real-space images of surface 
structures at the atomic level but the two techniques are really almost complementary. Scanning probe microscopy allows fast data acquisition and interpretation and the study of individual features, regardless of the degree of order, but it cannot deliver direct structural information about the three-dimensional arrangement of atoms at the pm level, in particular not for atoms below the outer-most surface layer. LEED can deliver precise crystallographic data but is restricted to relatively well-ordered surface structures. The results always reflect the properties of a large ensemble of surface unit cells. Photoelectron diffraction (PhD) [Wood07] and surface X-ray diffraction (SXRD) [Feid89] are related surface sensitive electron and X-ray diffraction methods, which deliver crystallographic information at a similar level of accuracy. PhD is element specific and does not require long-range order; SXRD also works under high-pressure conditions where electrons cannot be used. These methods, however, require synchrotron radiation and are therefore not as readily available as LEED systems, which are part of the standard equipment of most surface science laboratories.

In the previous sections we have highlighted only a small fraction of the research that can be carried out by LEED with an emphasis on simplicity in order to explain the basics of the technique. A number of recent innovations have opened up the technique to a variety of technically important surface and interface systems with relevance to biology and nano-electronics.

Much of recent developments in LEED-IV structure determination were directed towards improving the model calculations involved in the data analysis. This includes approximations that replace parts of the full quantum mechanical scattering calculations and thus speed up the optimization process (e.g. "Tensor LEED" [Rous93], "molecular T matrix approach" [Blan05]), "direct methods" aiming at a direct conversion of IV curves into a three-dimensional structure [Seub00], and better mathematical descriptions of scattering potentials and thermal vibrations of semiconductors and organic molecules. To date, computer power is only a limiting factor for very large unit cells with many ( $>20$ ) geometrical parameters to be optimized. The determination of a medium size structure can be performed on a modern personal computer within a matter of hours or a few days. Often the lack of enough experimental data for comparison with model calculations is a more severe limitation for the analysis of more complex surface structures with large unit cells. This limitation can be overcome by recording IV curves at different angles of incidence, each creating an additional set of data [Held95]. The sum of these improvements enables the accurate characterization of structures at the interfaces between inorganic substrates and large organic molecules as they are found in biological interfaces or organic electronic devices, and thus open exciting new applications for surface and interface crystallography by LEED.

Another exciting perspective is offered by the low-energy electron microscope (LEEM), a combination of imaging electron microscope and LEED. This microscopic technique has been developed by Bauer and Telieps already in the 1960's and 70's [Baue94,Baue98] but has become widely available only in the last decade or so. The combination of imaging and diffraction allows characterizing surface areas of the size of $\mu \mathrm{m}$ to $\mathrm{nm}$.
One application, often referred to as "micro-LEED", is the collection of LEED-IV data from an area of a few $\mu \mathrm{m}$ in diameter or less. This way, surface structures of single domains on single crystal surfaces [Figu06], artificial nanostructures of semiconductor devices, or crystallites of polycrystalline material [Corn10] can be determined, which enables surface structure determination for completely new classes of materials with a wide range of applications.

\section{REFERENCES}

[Baue94] E. Bauer, Rep. Prog. Phys. 57 (1994) 895.

[Baue98] E. Bauer, Surf. Rev. Lett. 5 (1998) 1275.

[Blan05] M. Blanco-Rey, P. de Andres, G. Held, D.A. King, Surf. Sci. 579 (2005) 89 .

[Brau05a] W. Braun, H.-P. Steinrück, G. Held, Surf. Sci. 575 (2005) 343.

[Brau05b] W. Braun, G. Held, Surf. Sci. 594 (2005) 203.

[Clar85] L. J. Clarke, „Surface Crystallography - An Introduction to Low Energy Electron Diffraction“, Wiley, Chichester, 1985.

[Corn10] A. Cornish, T. Eralp, A. Shavorskiy, R. A. Bennett, G. Held, S. A. Cavill, A. Potenza, H. Marchetto, S. S. Dhesi, Phys. Rev. B 81, (2010) 085403.

[Davi27] C. Davisson, L. H. Germer, Phys. Rev. 30 (1927), 705.

[Feid89] R. Feidenhans'l, Surf. Sci. Rep. 10 (1989) 105-188.

[Ferr04] N. Ferralis, K. Pussi, E. J. Cox, M. Gierer, J. Ledieu, I. R. Fisher, C. J. Jenks, M. Lindroos, R. McGrath, and R. D. Diehl, Phys. Rev. B 69 (2004) 153404.

[Figu06] J. de la Figuera, J.M. Puerta, J.I. Cerda, F. El Gabaly, K.F. McCarty, Surf. Sci. 600 (2006) L105.

[Hein91] K. Heinz, U. Starke, F. Bothe, Surf. Sci. Lett. 243 (1991) L70.

[Hein92] K. Heinz, U. Starke, M. A. Van Hove, G. A. Somorjai, Surf. Sci. 261 (1992) 57.

[Hein94] K. Heinz, Surf. Sci. 299/300 (1994) 433.

[Hein99] K. Heinz, S. Müller, L. Hammer, J. Phys.: Condens. Matter 11 (1999) 9437.

[Held95] G. Held, A. Wander, and D. A. King, Phys. Rev. B 51 (1995) 17856.

[Held98] G. Held, J. Schuler, W. Sklarek, and H.-P. Steinrück, Surf. Sci. 398 (1998) 154.

[Held01] G. Held, W. Braun, H.-P. Steinrück, S. Yamagishi, S. J. Jenkins, and D. A. King, Phys. Rev. Lett. 87 (2001) 216102.

[Henz77] M. Henzler in „Electron Spectroscopy for Surface Analysis“, Ed H. Ibach, Springer Berlin, 1977, 117.

[Henz91] M. Henzler, W. Göpel, „Oberflächenphysik des Festkörpers“, Teubner, Stuttgart, 1991.

[Li_09] H. I. Li, K. Pussi, K. J. Hanna, L.-L. Wang, D. D. Johnson, H.-P. Cheng, H. Shin, S. Curtarolo, W. Moritz, J. A. Smerdon, R. McGrath, and R. D. Diehl, Phys. Rev. Lett. 103 (2009) 056101.

[Mori10] W. Moritz, B. Wang, M.-L. Bocquet, T. Brugger, T. Greber, J. Wintterlin, and S. Günther, Phys. Rev. Lett. 104 (2010) 136102.

[Over98] H. Over, Prog. Surf. Sci. 58 (1998) 249.

[Pend74] J. B. Pendry, „Low Energy Electron Diffraction“, Academic Press, London, 1974.

[Pend80] J. B. Pendry, J. Phys. C 13 (1980) 937.

[Poon 04] H.C. Poon, M. Weinert, D.K. Saldin, D. Stacchiola, T. Zheng, W.T. Tysoe, Phys. Rev. B 69 (2004) 035401.

[Rous93] P. J. Rous, Prog. Surf. Sci. 39 (1992) 3.

[Seub00] A. Seubert, D. K. Saldin, J. Bernhardt, U. Starke K. Heinz, J. Phys. Condens. Matter 12 (2000) 5527.

[SSD_02] P. R. Watson, M. A. Van Hove, K. Hermann NIST Surface Structure Database: Version 5.0 (2002).

[Vanh79] M. A. Van Hove and S. Y. Tong, „Surface Crystallography by LEED“, Springer, Berlin, 1979.

[Vanh86] M. A. Van Hove, W. H. Weinberg, C.-M. Chan „Low-Energy Electron Diffraction“, Springer, Berlin, 1986.

[Woll98] J. Wollschläger, F. Schäfer, K.M. Schröder, Surf. Sci. 396 (1998) 94

[Wood64] E. A. Wood, J. Appl. Phys. 35 (1964) 1306.

[Wood07] D. P. Woodruff, Surf. Sci. Rep. 62 (2007) 1. 